\title{
Expertise anthropologique et politiques foncières
} au Sud

Anthropological expertise and land policies in the South

\section{Philippe Lavigne Delville et Pierre-Yves Le Meur}

\section{(2) OpenEdition}

\section{Édition électronique}

URL : http://journals.openedition.org/economierurale/4932

DOI : $10.4000 /$ economierurale.4932

ISSN : 2105-2581

\section{Éditeur}

Société Française d'Économie Rurale (SFER)

\section{Édition imprimée}

Date de publication : 30 juin 2016

Pagination : 81-94

ISSN : 0013-0559

\section{Référence électronique}

Philippe Lavigne Delville et Pierre-Yves Le Meur, «Expertise anthropologique et politiques foncières au Sud », Économie rurale [En ligne], 353-354 | mai-juillet 2016, mis en ligne le 30 juin 2018, consulté le 19 avril 2019. URL : http://journals.openedition.org/economierurale/4932 ; DOI : 10.4000/ economierurale.4932 


\title{
Expertise anthropologique et politiques foncières au Sud
}

\author{
Philippe LAVIGNE DELVILLE •IRD, UMR GRED, Montpellier \\ philippe.lavignedelville@ird.fr. \\ Pierre-Yves LE MEUR • IRD, UMR GRED, Montpellier \\ pierre-yves.lemeur@ird.fr.
}

\begin{abstract}
Les savoirs anthropologiques mobilisés dans les politiques foncières au Sud croisent des concepts devenus obsolètes et des récits de politique publique discutables. La forte incertitude épistémologique et politique qui pèse sur les politiques foncières et la multiplication de sites de production des savoirs fonciers poussent à concevoir l'expertise comme alimentation de réseaux et mise en débats des connaissances plus que comme une réponse à des commandes. Mobilisant leur expérience d'anthropologues, de chercheurs et de praticiens de l'expertise, les auteurs montrent comment l'expertise anthropologique peut à la fois éclairer les conceptions et les effets des politiques foncières tout en s'engageant dans des formes d'accompagnement de ces politiques assumant la structurelle incomplétude de l'action publique.
\end{abstract}

MOTS-CLÉS : anthropologie, expertise, politiques foncières, politiques publiques, approche cognitive

\section{Anthropological expertise and land policies in the South}

Anthropologists involved in land policies in the South confront themselves to concepts that have once been central to their discipline but are obsolete nowadays, and to debatable policy narratives. Given the current epistemological and political uncertainty characterizing land policies and the nurturing of knowledge production sites on land issues, expertise in this field can be seen as feeding policy networks and promoting debate on land knowledge than as responding to fixed terms of references. Drawing on our experience as anthropologists involved in research and expertise, we show to what extent anthropological expertise contributes to unveil assumptions underlying land policy debates while accompanying these policies and assuming the structural incompleteness of public action. (JEL: Q15, Z13).

KEYWORDS: anthropology, expertise, public policies, land policies, cognitive approach

a relation entre connaissance et action ne

va pas de soi. Toute politique publique repose sur des opérations de « réduction de la complexité du réel » (Muller, 1990), en fonction de référentiels cognitifs, d'intérêts, de logiques institutionnelles. Les argumentaires sur lesquels reposent les politiques ou les propositions visant à les réformer prennent la forme de récits causaux (Roe, 1994), dans une logique de dramatisation des problèmes et de légitimation des solutions, et ont donc une forte dimension rhétorique et argumentative (Apthorpe, 1997). Définir une politique suppose des arbitrages, des choix, selon des modalités qui ne relèvent pas de l'analyse scientifique mais bien plus de rapports de force autour de la relation complexe entre définition des problèmes, identification des solutions et mise en action des choix effectués (Zittoun, 2013).

Le foncier est un objet complexe, partie intégrante des relations de production et d'échanges, de la construction des appartenances et des rapports État/société. Il pose des questions de politique publique sectorielle (policy), mais aussi d'enjeux de pouvoirs (politics) et de choix de société 
(polity) (Leca, 2012). La majorité des pays du Sud connaît 1) un dualisme légal d'origine coloniale entre un cadre légal fondé sur la propriété privée et une part plus ou moins importante du territoire relevant de situations «extra-légales »; 2) une forte diversité des rapports fonciers locaux, à la croisée de modes d'exploitation du milieu, de normes sociales et d'histoires économiques et politiques spécifiques, à la fois précoloniales, coloniales et postcoloniales.

Orientées vers la promotion du marché foncier au XIX ${ }^{\mathrm{e}}$ siècle, les politiques foncières ont mis en avant les redistributions foncières et les réformes agraires au cours du $\mathrm{XX}^{\mathrm{e}}$ siècle. Avec la chute du mur de Berlin, la question de la répartition des terres et des inégalités a été évacuée au profit de celle de la reconnaissance légale des droits locaux (jusqu'ici informels ou extralégaux), dans une tension entre logique de sécurisation des modes populaires d'accès à la terre et logique de promotion de la compétition et du marché. Socialement mais aussi scientifiquement contestées, les logiques de privatisation sont très prégnantes dans les discours politiques et les pratiques des États et de l'industrie de l'aide. Les discours sur la lutte contre la pauvreté et sur la reconnaissance des droits autochtones se heurtent aux discours de promotion de la propriété privée et du marché. Incertains dans leurs objectifs et leurs finalités, objets de forts enjeux politiques et économiques, les processus de réforme foncière dans les pays du Sud sont soumis à des prescriptions internationales contradictoires.

La «demande sociale» actuelle pour une expertise en sciences sociales prend place dans ce contexte particulièrement controversé et mouvant. Anthropologues, chercheurs statutaires au sein d'un institut de recherche public spécialisé dans le champ du développement, nos trajectoires antérieures nous ont amenés à pratiquer l'expertise et à entrer dans des démarches de recherche appliquée sur les politiques foncières rurales, dans différents contextes professionnels, institutionnels et géographiques (Afrique de l'Ouest, NouvelleCalédonie, Asie du Sud-Est). Nous partirons des transformations contemporaines de la figure de l'expert pour ensuite aborder le rapport à l'empirie des politiques foncières, qui renvoie, entre autres, au lien entre la recherche et l'expertise, et à la place des questionnements de sciences sociales face à un objet largement « préempté » par le droit, la géomatique, l'économie. Nous approfondirons ensuite la question des liens cognitifs ou épistémologiques entre sciences sociales et politiques foncières, ainsi que la façon dont des catégories issues des sciences sociales sont mobilisées et réinterprétées. Enfin, nous discuterons le rôle de l'expertise, en tant que champ de mobilisation des catégories et des acteurs de la recherche en sciences sociales, marqué par le pluralisme des producteurs et des demandeurs de savoirs sur le foncier. Nous nous intéresserons en particulier à la position institutionnelle de l'expertise, puis aux modalités de son insertion dans les trajectoires des politiques foncières.

\section{L'expert Une figure en mutation}

L'expertise est la «production d'une connaissance spécifique pour l'action» (Lascoumes, 2002b), « une activité particulière d'exercice diagnostique du savoir en situation problématique, dans le cadre d'une mission intégrée à un processus décisionnel dont l'expert n'est pas le maître » (Théry, 2005). Isabelle Théry (2005) distingue trois types d'expertise :

- l'expertise de service, où le mandataire a ses compétences propres, mais demande une expertise technique sur un domaine qu'il ne maîtrise pas. Nous sommes ici dans le modèle de l'expertise technique et judiciaire, où l'expert est censé être neutre et mobiliser des 
savoirs décontextualisés pour éclairer la décision ;

- l'expertise de consensus, qui se déroule en général au sein de commissions, et dont le rôle est d'établir des normes à partir d'un débat entre experts de différentes spécialités. «La spécificité de ce type d'expertise est d'organiser procéduralement la confrontation et l'ajustement entre des acteurs aux compétences différentes » afin de permettre « l'élaboration même d'un consensus éclairé, sur des questions où les savoirs sont en cours d'élaboration, et les enjeux éthiques et politiques complexes »;

- enfin, l'expertise d'engagement, où « un spécialiste est mandaté à la fois pour proposer une analyse scientifique, établir un diagnostic et s'engager sur des propositions pour l'action (réformes juridiques, réformes des politiques publiques) ».

La première forme renvoie au modèle classique où l'État mobilise l'expertise interne de son administration ou recourt à des acteurs spécialisés, dans la recherche ou les institutions finalisées. Il repose sur un monopole du savoir légitime aux mains des experts et des politiques, excluant l'expérience pratique des acteurs concernés par un sujet, leurs connaissances, leurs savoirs (d'usage, vicinaux, etc.). À partir des années 1980, la remise en cause de la « double délégation » (Callon et al., 2001) $\mathrm{du}$ pouvoir aux politiques et aux experts réintroduit le «public» dans les controverses, ce qui entraîne une profonde redéfinition de l'expertise. L'expert n'est plus tant celui qui apporte une connaissance prédéfinie - selon des problématisations et des catégorisations qui sont les siennes ou celles de l'État - que celui qui favorise l'exploration des controverses et la production collective d'une analyse (Lascoumes, 2002a). Cette évolution va de pair avec la perte de monopole de l'expert institué et la reconnaissance de «l'expertise quotidienne » des acteurs (Sennett, 2009). Les associations, les mouvements sociaux s'emparent de l'expertise, qui devient une forme de mobilisation (Delmas, 2011).

La troisième forme se situe dans un espace que l'on peut qualifier de pré- ou paradécisionnel. Elle correspond à la qualification que propose Philippe Roqueplo (1997) de l'expertise comme transgression; une transgression qui pousse le chercheur aux frontières de son champ de compétence, dans une logique d'engagement. La mobilisation de l'expert se situe ainsi dans une tension entre mobilisation de savoirs spécifiques et montée en généralité, neutralité axiologique et implication politique. Elle prend forme entre réponse à des commandes prédéfinies et problématisation autonome, entre mobilisation de connaissances propres et animation de réflexions collectives, dans des contextes où les acteurs mobilisés dans les débats de politique publique se diversifient, où les lieux de production de connaissances se multiplient, avec des productions «hybrides » entre discours scientifique et discours politique.

\section{Recherche et expertise sur/dans les politiques foncières : enjeux cognitifs et politiques}

\section{Le problème des rapports connaissance/action dans les politiques publiques}

Une politique publique est un ensemble d'actions et d'inactions, visant à organiser un secteur de la société. C'est le produit d'une histoire institutionnelle (Dubois, 2003) qui se sédimente dans des institutions prises en charge par certains groupes professionnels. Elle s'inscrit de manière plus ou moins explicite dans un référentiel global ${ }^{1}$ (Muller, 1990) à travers une série de désajustements/réajustements.

1. Nous prenons ici le terme «référentiel» dans une acception plus large que Muller. Sur les débats autour de la notion, $c f$. Faure et al. (1995). 
Elle prend forme à travers un ensemble de catégories de pensées et d'instruments (Lascoumes et Simard, 2011). Elle se concrétise à travers une succession de réinterprétations et une mobilisation sélective des instruments par les différents acteurs concernés, aux différents niveaux de la mise en œuvre. Les politiques sont plus souvent un ensemble complexe et ambigu, marqué par des objectifs peu clairs et contradictoires (Lascoumes et Le Galès, 2007), une mise en œuvre incertaine (Pressman et Wildavsky, 1973), de forts sentiers de dépendance institutionnelle, dans des rapports complexes avec le jeu politique (Leca, 2012). Une politique stabilise à la fois des façons de penser le secteur et ses problèmes, une hiérarchie entre acteurs et groupes professionnels légitimes pour en traiter, et un certain nombre d'institutions qui la prennent en charge (parfois en concurrence [Gaxie, 1997]). C'est une question d'idées, d'intérêts et d'institutions (Palier et Surel, 2005), mais aussi d'acteurs, d'alliances, de conflits, d'évitements et de malentendus.

La pression à agir sur les gouvernants résulte de processus complexes et contingents de problématisation et de publicisation, par lesquels un problème, porté par certains acteurs, est construit comme problème public nécessitant une prise en charge (Cefaï, 1996 ; Hassenteufel, 2010). La formulation du problème relève de récits causaux, éventuellement concurrents, et est l'objet de luttes de définition (Zittoun, 2013), autour des contours du problème, de ses causes, de ses responsables, et des réponses à apporter. La construction des problèmes, celle des solutions, l'élaboration des « récits » raccordant les deux (en partant de la solution ou du problème), et légitimant la nécessité d'agir ou de légitimer/rationaliser dans un sens donné, se fait à travers des interactions au sein et entre « réseaux de politique publique », forums et arènes (Jobert, 1998).
Tout en mobilisant des catégories scientifiques ou des travaux de recherche, les récits de politique publique sont donc des construits sociaux à finalité argumentative, et non pas des énoncés scientifiques (Roe, 1994). Toute intervention publique repose sur des catégories simplifiées. Certes, les réseaux de politiques publiques et la médiatisation mobilisent, à des degrés variables des énoncés et des analyses issues de la recherche, mais ceux-ci sont rarement la principale source de savoirs. Des experts, des médiateurs, parfois les chercheurs euxmêmes, jouent un rôle de traduction et de réinterprétation des énoncés scientifiques en énoncés de politique publique.

\section{Le foncier, un objet complexe aux forts enjeux cognitifs et politiques}

Le foncier est l'ensemble des rapports qu'entretiennent les hommes entre eux à propos de l'accès et du contrôle de la terre et des ressources naturelles. La façon dont une société définit les droits de propriété (au sens large de property rights) traduit sa conception des rapports entre société et nature, entre individus et collectifs sociaux, entre État, pouvoirs locaux et citoyens. Le foncier concentre des enjeux économiques évidents au sens où la terre et les ressources naturelles sont une base importante de l'économie et où les inégalités d'accès à ces ressources se traduisent dans les inégalités économiques. C'est aussi un rapport politique, à différents niveaux (Boone, 2007 ; Jacob et Le Meur, 2010).

Nombre de pays du Sud connaissent un dualisme juridique, issu d'un legs colonial, où la propriété privée, en pratique réservée à une petite élite, coexiste avec de larges terres « publiques », sous contrôle étatique, mais en pratique appropriées et occupées par les sociétés locales. Du fait de ces rapports historiquement complexes entre sociétés locales et État, et de la complexité des processus de changement social, le foncier dans les pays en développement est un 
« champ social semi-autonome » (Moore, 1973), en partie régi par des normes locales relevant d'autres répertoires de sens que les normes étatiques. Caractéristique structurelle de ces sociétés, la pluralité des normes a des effets variés selon le degré d'autonomie du local ; liée à la pluralité des arènes de négociation et de résolution des conflits, elle favorise les stratégies d'acteurs et la concurrence entre institutions de régulation foncière (Lund, 2002), et in fine la politisation des rapports fonciers.

L'organisation des migrations, le développement de filières agricoles, le contrôle social de certaines populations, la redistribution clientéliste de l'accès à la terre, les alliances politiques avec les pouvoirs fonciers locaux, la captation de la terre au profit de l'élite politico-économique font partie des politiques poursuivies par les États. Dans un contexte de financiarisation des marchés des matières premières, les États sont partie prenante des politiques d'accaparement foncier, en faveur des élites nationales et/ou d'investisseurs internationaux (Borras et al., 2011).

\section{Cadres cognitifs et savoirs anthropologiques dans les débats de politiques foncières}

Les sciences sociales cherchent à produire les cadres conceptuels et analytiques permettant de décrypter les pratiques et les représentations sociales. La mise au jour des cadres cognitifs mobilisés dans les récits de politique publique, et donc l'analyse de la façon de poser les problèmes, vus à un certain moment comme enjeux politiques, est une des sources de tensions entre chercheurs en sciences sociales et responsables des politiques foncières, en amont même des recherches localisées sur les dynamiques foncières. Les récits de politiques publiques reposent en effet fréquemment 1) sur des catégories analytiques (propriété, droit, marché, communauté) discutables d'un point de vue des sciences sociales, voire obsolètes ;2) sur des récits simplifiés, normatifs et empiriquement contestables.

\section{Catégories scientifiques, catégories politiques}

Pour les sciences sociales, un droit est « une action socialement autorisée » (Alchian et Demsetz, 1973). Les droits de propriété sont ainsi des prérogatives, détenues par des individus ou des groupes sociaux, leur autorisant certaines actions en échange d'obligations sociales. Ils prennent la forme de «faisceaux de droits » plus ou moins étendus, relevant d'un ensemble de normes sociales qui les légitiment, et sont garantis par des autorités. Ces droits de propriété portent à la fois sur la terre et les ressources naturelles renouvelables ; ils combinent des «droits opérationnels », portant sur les usages possibles de la terre ou des ressources naturelles concernées, et des «droits d'administration », qui organisent la répartition et la transmission des droits opérationnels (Colin, 2008). Une telle perspective se heurte d'abord aux conceptions des droits de propriété en économie et en droit standard, qui les considèrent non pas comme un rapport social, mais comme un rapport entre les hommes et les choses et les assimilent facilement à la seule propriété privée. Elle se heurte ensuite aux conceptions de la propriété, promues dans les discours de politique foncière, qui varient selon les contextes entre définitions juridiquement rigides et acceptions molles.

Les discours de politique foncière sur les sociétés rurales oscillent entre une représentation considérant que le marché et la propriété individuelle sont déjà généralisés, et niant les régulations collectives existantes, et à l'inverse, une représentation qui surestime le rôle de la « coutume », de la «tradition ». Bien que les notions, autrefois clés pour l'anthropologie, de lignage, communauté, village, coutume, etc., aient été largement « déconstruites » 
depuis (cf. Kuper, 1982 pour le lignage), nous observons fréquemment un usage réifié et une résilience politique de catégories obsolètes d'un point de vue scientifique, ce qui constitue l'un des problèmes majeurs que doivent affronter les chercheurs en sciences sociales qui s'impliquent dans le champ des politiques publiques et tentent de restituer une vision réaliste des rapports sociaux et fonciers. Alors que les anthropologues ont montré à quel point la logique des systèmes coutumiers, marquée par la fluidité, la négociabilité et une oralité partielle, était profondément politique (Chauveau, 1998), ils doivent en quelque sorte se battre contre les fantômes de leur propre passé disciplinaire.

La trajectoire de ces catégories dans les champs du politique et du développement montre que leur résilience n'est pas simple survie mais transformation. Le terme de coutume est revenu au-devant de la scène, à la fois dans le cadre d'opérations de reconnaissances de droits locaux et dans le cadre des politiques foncières postcoloniales dans le Pacifique (Filer, 2006). La coutume a historiquement constitué une source du droit, via un processus normatif de sélection, que ce soit dans le cadre de la construction de l'État absolutiste en Europe ou des administrations coloniales (Stamm, 2013). Elle est ainsi devenue le référentiel (souvent fictif) d'un dispositif institutionnel d'origine coloniale, basé sur la codification des droits, des statuts, des autorités : une affaire de gouvernance.

La notion de communauté a également une généalogie complexe, en particulier coloniale, qui a été réactualisée via le discours sur les modes de gestion «communautaire» des ressources naturelles, basés sur l'idée, souvent implicite, selon laquelle il y aurait une entité - une « communauté »- préexistante à l'intervention publique qui constituerait son objet et sa « cible ». La notion de communauté a traversé les époques coloniales et postcoloniales, nimbée d'un flou définitionnel qui en fait sa force politique. Les « communautés locales » présupposées et visées par les interventions correspondent à des regroupements sociaux très hétérogènes du point de vue de la composition, de l'origine, de la taille, de l'institutionnalisation. Or les politiques foncières et de gestion des ressources naturelles sont en général aveugles à ces différences et surtout au fait qu'elles sont elles-mêmes productrices de «communautés » par injection de normes, de ressources, de frontières nouvelles dans les sociétés cibles de leur ingénierie sociale (Li, 2007).

\section{Référentiels, cadrages et récits de politiques}

La survie politique de concepts obsolètes en sciences sociales alimente (et se nourrit de) la prégnance de récits de politique publique très puissants, dont deux irriguent abondamment les politiques foncières : la théorie de la modernisation et la théorie évolutionniste des droits de propriété (voir respectivement Kitching, 1982 ; Platteau, 1996) $)^{2}$.

Théorie de la modernisation et théorie évolutionniste des droits de propriété partagent une conception téléologique et normative des processus de changement social, dans laquelle les sociétés doivent nécessairement passer d'un stade initial mal défini, mais qualifié de «traditionnel », et relevant de conceptions communautaires de la société et collectives du foncier - à une modernité définie comme la situation des sociétés industrialisées, donc individualiste et structurée autour du

2. S'opposant à ces récits, le récit «communautaire » surestime à l'inverse la cohérence et l'effectivité des régulations coutumières, à partir d'une conception organiciste des «communautés ». Finalement tout aussi normatif et a-historique, il légitime des formes de « retraditionnalisation » du foncier et de réhabilitation des autorités coutumières. 
marché. Selon les auteurs, ce changement se fait spontanément, sous l'influence de la démographie, du marché, de la recherche de profit, ou bien nécessite des interventions étatiques pour imposer, voire accompagner, ces évolutions. La théorie évolutionniste des droits de propriété relève de cette logique (Platteau, 1996), y compris dans sa variante contemporaine de "l'innovation institutionnelle induite ». Selon celle-ci, les droits fonciers coutumiers évoluent spontanément sous l'influence de la démographie et du marché, mais les processus d'individualisation et de marchandisation suscitent des différends sur les normes, des flous sur les droits, des conflits, et donc une demande pour des interventions étatiques visant à formaliser les droits de propriété privée, achevant ainsi le basculement dans une propriété privée individuelle.

Nulle part, pas même en Europe censée être le «modèle », les trajectoires foncières n'ont pris cette forme (Béaur et al., 2013 ; Lavigne Delville et Karsenty, 1998). L'analyse contextualisée des trajectoires socio-foncières montre, au contraire, des rapports fonciers profondément enchâssés dans les rapports sociaux et politiques, combinant prérogatives individuelles et régulations collectives, et des processus d'individualisation mais aussi de marchandisation contingents éventuellement réversibles (Colin et Woodhouse, 2010).

Le récit évolutionniste se couple avec un récit séduisant mais souvent démenti dans les faits, sur les liens entre droits de propriété et développement économique. Selon ce récit, la délivrance de titres de propriété privée sécurise les producteurs, leur donne accès au crédit (ils peuvent utiliser leur titre comme garantie); sûrs de bénéficier du fruit de leurs efforts, pouvant mobiliser du capital, ils vont investir et accroître la productivité de la terre, le marché foncier se chargeant parallèlement de faire circuler la terre vers les producteurs les plus efficaces. Là encore, la recherche économique a, de longue date, démonté ce récit (Platteau, 1996) : il suppose une équivalence fausse entre formalité des droits et sécurité foncière et surestime l'insécurité foncière en situation «informelle » (Lavigne Delville, 2006) ; il voit dans l'informalité la source principale des conflits, alors que la pluralité des normes et la concurrence entre autorités pour arbitrer les conflits sont la cause majeure d'une insécurité institutionnelle générée par la possibilité de «rouvrir un dossier » dans une autre arène en fonction des résultats attendus. Il suppose un système bancaire développé, présent en milieu rural, prêt à prêter aux agriculteurs, ce qui est rarement le cas.

Malgré ces démentis tant théoriques qu'empiriques, le récit sur la formalisation des droits comme levier de développement économique est régulièrement invoqué dans les débats de politique foncière. Il est régulièrement relancé par les institutions internationales, sous des formes actualisées : ainsi la promotion de l'ouvrage de Hernando de Soto (2005) Le mystère du capital correspond-elle à une stratégie de relégitimation de la formalisation des droits en termes de lutte contre la pauvreté.

Les chercheurs en sciences sociales sollicités pour une expertise sont confrontés aux enjeux politiques structurant la question foncière, aux concepts, aux cadrages conceptuels mobilisés par les commanditaires, et ils doivent gérer une certaine difficulté à assumer la simplification des énoncés de politique publique.

\section{Intervenir comme expert Entre dévoilement et accompagnement}

L'expertise foncière en sciences sociales suit pour partie les évolutions générales de l'expertise décrites au début de cet article, mais dans le cadre des luttes de définition 
et des rapports de force spécifiques à la question foncière. La mobilisation d'expertise demeure majoritairement le fait des États ainsi que des institutions d'aide. Avec le tournant néolibéral, la «participation » aux politiques publiques est devenue un mot d'ordre et un passage obligé mais, dans les pays sous régime d'aide (Chauveau et Lavigne Delville, 2013), elle prend des formes très variées, souvent plus instrumentales que délibératives (Blondiaux et Sintomer, 2009). Pourtant, une demande d'expertise émerge aussi du côté des organisations de la société civile. Par ailleurs, la recherche académique et l'expertise ne sont pas les seuls modes de production de connaissances : les institutions d'aide, ONG, think tanks, produisent des savoirs hybrides ; des mouvements sociaux s'emparent parfois de la thématique. Les uns et les autres peuvent solliciter des chercheurs, pour contribuer à leurs études ou alimenter leur réflexion.

\section{La pratique de l'expertise Des configurations variées}

Les modalités et le contenu de la demande d'expertise dépendent du commanditaire comme des configurations dans lesquelles elle s'inscrit. Selon les cas, les interlocuteurs seront un public élargi ou bien un cercle restreint de commanditaires proche des lieux de décision. L'apport demandé à l'expert pourra relever :

- d'une expertise de service, à travers la mise à disposition de cadres conceptuels ou de connaissances préexistantes ;

- d'une recherche appliquée, à travers la production de connaissances originales sur des questions spécifiques ;

- d'une expertise d'engagement, par des apports à forte visée appliquée, proposant des options politiques ou des stratégies opérationnelles.

La mobilisation d'expertise se fait en général au sein de «communautés épistémiques » déjà plus ou moins stabilisées, ou du moins, de « coalitions discursives » pouvant «parfaitement réunir des acteurs qui ont des vues contraires des intérêts qu'ils entendent promouvoir, mais qui s'accordent sur le cadre cognitif et les institutions pour la gestion de leurs conflits » (Jobert, 2004). Toutefois, le chercheur intervenant comme expert confronte ses cadres conceptuels et ses connaissances scientifiques à d'autres questionnements, connaissances et acteurs. Le travail réalisé en Nouvelle-Calédonie en accompagnement de la réforme foncière illustre bien ce dialogue au cours duquel l'expert apporte des outils et des savoirs issus d'autres contextes fonciers à des agents de la réforme qui sont, quant à eux, les experts de la situation (Le Meur, 2011a). La connaissance produite est donc toujours construite et négociée dans le cours de la situation d'expertise, quoiqu'à des degrés très variables selon les configurations.

Le cadre politique de l'expertise dépend des configurations d'acteurs, de la nature des controverses autour de la politique foncière, du degré de polarisation des débats. Il dépend aussi de la séquence de politique concernée : formulation, mise en œuvre, évaluation ${ }^{3}$.

L'ouverture des acteurs aux questionnements et aux savoirs de sciences sociales, qui influence l'autonomie laissée à l'expert, dépend donc largement des configurations. Une part significative de la demande relève de l'expertise de service, de la part des États ou des agences d'aide, autour de questionnements prédéfinis. Une telle configuration laisse peu de place à la prise au sérieux des dynamiques foncières

3. Aucune politique réelle ne suit parfaitement le cheminement linéaire allant du diagnostic à la mise en œuvre et à l'évaluation (Massardier, 2003). La réforme foncière du Bénin montre ainsi un télescopage du temps des choix politiques et de celui de la mise en œuvre, aboutissant à des contradictions de temporalités et une confusion sur les objectifs et les cadres institutionnels (Lavigne Delville, 2010). 
et de leurs enjeux politiques, économiques et sociaux, pas plus qu'à des problématisations qui ne soient pas cadrées par les théories de la modernisation et par les conceptions $\mathrm{du}$ foncier portées par les corps professionnels des géomètres et des juristes. Or, dans une phase d'interrogation sur les politiques en place et sur les alternatives possibles, l'enjeu pour des chercheurs est au contraire de mobiliser des cadres conceptuels ainsi que des connaissances solides, ce qui suppose, à un degré ou un autre, de questionner la commande et ses présupposés, mais aussi de contribuer à alimenter un débat public autour des diagnostics et des enjeux.

Les situations d'expertise que nous avons expérimentées sont diverses. Nos deux principales expériences (Bénin et Nouvelle-Calédonie) relèvent de formes hybrides d'expertise d'engagement au sens de Théry, marquées par une imbrication forte entre mécanismes de production de savoirs et de prises de décisions (voir aussi Lascoumes, 2002b).

\section{Entre dévoilement et accompagnement}

François de Singly (2002) identifie deux fonctions de la sociologie, une fonction de dévoilement (qui met au jour les rapports de domination, les argumentaires de légitimation, les effets pervers d'une politique, etc.) et une fonction d'accompagnement, qui permet aux acteurs de mieux penser leur situation. Dans cette conception, l'utilité de la recherche en sciences sociales pour les acteurs sociaux est double :

- elle offre un regard construit et distancié sur leurs pratiques, leurs fondements, leurs enjeux et leurs effets ;

- elle leur permet, par des médiations diverses, de mieux comprendre le monde, leur propre position, les déterminations qu'ils subissent, les logiques globales auxquelles ils participent, leur donnant des outils pour s'en distancier ou lutter contre.
Cette double dimension vaut aussi pour l'expertise sur le foncier. Dès lors qu'elle ne se limite pas à une réponse formatée à un questionnement lui-même formaté, elle participe de la construction des cadrages - ou de leur questionnement. Le travail d'expertise s'inscrit ainsi dans un dialogue critique avec les responsables des politiques foncières, les acteurs de l'administration foncière, les commanditaires. Dans sa dimension de «dévoilement», en complément et en articulation avec des recherches fondamentales, l'expertise produit des connaissances, propose une lecture construite des dynamiques sociofoncières, met en perspective les débats et les controverses, contribue à identifier les enjeux des politiques. Mais les analyses et connaissances socio-anthropologiques se diffusent difficilement, tant pour des raisons cognitives (cf. ci-dessus) que parce qu'elles mettent en cause des cadrages et donc des intérêts. Face à «l'incorporation malaisée du savoir anthropologique à l'action sociale », Baré (1995) considère qu'un travail spécifique «d'accompagnement de l'information anthropologique » est nécessaire pour que des connaissances utiles soient utilisables (et si possible utilisées). Dans sa dimension d'accompagnement, l'expertise permet aux acteurs concernés ${ }^{4}$ - pour autant qu'ils l'acceptent - de déplacer leurs questionnements, de prendre leur distance par rapport à leurs catégories d'analyse, d'enrichir leur connaissance du secteur et de ses dynamiques ${ }^{5}$.

4. Des restitutions limitées aux commanditaires et des clauses de confidentialités limitent trop souvent la restitution et la mise en débat élargi des résultats d'expertise (qui pourraient pourtant être considérés comme des biens publics car financés par l'argent public) et ne permettent pas d'alimenter de la même façon la réflexion de l'ensemble des parties prenantes.

5. Ce double rôle vaut aussi pour les expertises commanditées par des think tanks ou des mouvements sociaux, qui y chercheraient des outils pour affiner leur critique des politiques en cours ou en débat et élaborer un positionnement et des propositions propres. 
Le travail effectué avec l'agence chargée de la réforme foncière en NouvelleCalédonie (Le Meur, 2010, 2011a) illustre bien ce processus, en particulier du fait de son inscription dans le temps. Les missions se sont échelonnées sur plusieurs années, les thèmes en étant définis dans une logique de dialogue entre expert et agence et in fine d'accompagnement mutuel : mission exploratoire sur le débat du « cadastre coutumier », réflexion sur les modes d'objectivation du savoir foncier accumulé au sein de l'agence, formation au diagnostic foncier, participation à la réflexion générale sur le régime d'administration foncière, appui méthodologique au bilan des 30 ans de la réforme foncière et au traitement de la revendication. Le travail mené avec l'agence ne s'est donc pas réduit à l'importation simple d'outils anthropologiques dans un contexte d'interaction avec un opérateur de politique publique : il était aussi affaire de mise en œuvre de ces ressources dans le cours même d'un travail d'expertise anthropologique qui s'est révélé collectif et réflexif.

Souvent, l'enjeu d'une expertise n'est pas tant dans la production de connaissances originales que dans la mise à disposition et la mise en débat d'un cadre d'analyse et de connaissances socio-anthropologiques, posant dans des termes plus justes la question des dynamiques foncières ou les enjeux des politiques, favorisant ainsi la traduction de ces savoirs dans les forums et arènes des politiques. Une telle traduction suppose nécessairement des reformulations et des simplifications, qui sont frustrantes pour le chercheur, mais n'en sont pas moins inévitables - voire quelque part nécessaires.

Dans le cas du travail réalisé au Bénin sur la réforme de la politique foncière rurale au début des années 2000 (Lavigne Delville, 2007, 2009, 2010, 2011; Le Meur, 2006, 2011b), la commande initiale portait sur une mission pluridisciplinaire de durée limitée, visant à définir un schéma d'actions pour la mise en œuvre de la future loi foncière. La renégociation des termes de référence a abouti à une refonte du processus, étalé sur neuf mois avec différentes étapes de diagnostic et d'élaboration concertée des propositions. L'expertise s'est inscrite dans une durée plus longue autorisant l'instauration d'un dialogue autour des outils mais aussi du cadre général de la future loi, selon une logique combinant là encore dévoilement, via les études spécifiques sur l'outil PFR, et accompagnement d'une réflexion visant à déplacer la focale de la politique foncière vers une logique moins technocratique de sécurisation foncière.

Le dialogue sur les problématiques et les questionnements, l'identification de « catégories d'interface » et de " raisonnements intermédiaires » (Lavigne Delville, 2007) permettant d'articuler les questionnements, les interactions régulières au cours de l'expertise, la pédagogie dans les restitutions, contribuent fortement à la recevabilité (toujours partielle et reformulée) des analyses issues de la recherche et de l'expertise, ce qui, encore une fois, plaide pour des processus d'interactions étalés dans le temps plus que pour des missions ponctuelles. La fonction d'accompagnement peut aller au-delà de la production de cadres d'analyse et de connaissances, lorsque les experts sont mobilisés pour contribuer à la définition des cadrages de la politique, à celle des stratégies de mise en œuvre, à la conception des outils, à la formation des équipes.

Pour autant qu'on la positionne au sein de réseaux élargis d'acteurs, et en termes de controverses entre communautés épistémiques, cette conception de l'expertise permet de rendre compte de la gamme des pratiques d'expertise, au sein d'une double tension structurelle, entre production et mise à disposition/traduction de connaissances, d'une part, entre réponse à 
des demandes (plus ou moins explicites) et engagement propre dans un travail de problématisation et de cadrage (Robert, 2008) d'autre part.

Une telle conception montre que le succès d'une expertise, dans sa capacité à influencer la décision, tient moins à la qualité des savoirs scientifiques mobilisés qu'à la capacité de l'expert à s'inscrire dans une communauté de politique publique, à travailler de façon active à la traduction des savoirs scientifiques, c'est-à-dire à la construction collective d'analyses, de cadrages et de récits de politique qui soient moins réducteurs ou prennent mieux en compte les positions et intérêts d'acteurs marginalisés.

$*$
$* \quad *$

\section{Sur le seuil de la caverne, parfois dedans}

L'expertise est, comme le souligne Dumoulin (2005), «une forme parmi d'autres de recours aux savoirs académiques dans la fabrique de l'action publique ». Elle pose la question de la critique et de la distance par rapport à une action publique inévitablement ambiguë et contradictoire, nécessairement marquée par des enjeux politiques et institutionnels (Lascoumes et Le Galès, 2007). Une expertise n'a pas d'impact par elle-même. La capacité des experts à enrichir les cadrages et à déplacer les problématisations dépend des configurations d'acteurs et des intérêts en jeu. Elle suppose, le plus souvent, de s'inscrire dans un réseau d'acteurs ayant des intérêts à mobiliser ces résultats dans les controverses de politiques publiques et les rapports de force.

De nombreux chercheurs académiques hésitent à s'affronter aux inévitables simplifications et aux risques d'instrumentalisation que comporte l'expertise. La position défendue ici consiste, dès lors qu'il semble exister une ouverture suffisante des réseaux de la décision ou que les risques justifient de se mobiliser, à accepter d'être partie prenante de processus de formulation ou de mise en œuvre des politiques foncières, tout en conservant une distance critique, alimentée par des recherches fondamentales. En articulant recherche fondamentale et expertise ou recherche appliquée, dans des configurations variées, en ne se limitant pas aux commandes mais en restituant ses travaux dans des forums variés, l'ambition du chercheur expert est d'alimenter la réflexion des différents types d'acteurs, de contribuer activement à la traduction entre types de connaissances et donc de prendre acte de la pluralité des sites et acteurs producteurs de savoirs. Il cherche à contribuer à la façon dont les problèmes sont posés et les solutions définies, à ouvrir le débat et ainsi contribuer à cette "démocratisation des connaissances et des choix » qui est pour Lascoumes (2002b) une des fonctions contemporaines de l'expertise. Il s'agit ainsi d'assumer, sous conditions, un engagement dans des processus actifs d' «accompagnement », dans des forums et parfois des arènes de débat sur les politiques foncières.

Discutant l'opposition classique entre une recherche externe, indépendante, distanciée, et une recherche au plus près des acteurs, de leurs savoirs, de leurs pratiques, Didier Fassin revisite l'idée platonicienne de la caverne. Réfutant l'opposition entre les deux postures, il se positionne « sur le seuil de la caverne, c'est-à-dire en alliant l'attention à l'égard des agents, de ce qu'ils disent, pensent et font, et le recul pour saisir ce qui leur échappe, soit qu'ils aient intérêt à ne pas voir, soit qu'ils occupent une place ne [le] leur permettant pas » (Fassin, 2012). Lorsqu'il franchit le seuil de la caverne le temps d'une expertise, le chercheur s'inscrit dans une logique d'engagement critique au sein de formes variées d'expertise et de participation à des forums. Cette posture vise à favoriser des évolutions des cadres cognitifs et des problématisations, et une conception plus 
réaliste de l'action publique, tout en assumant la nécessaire incomplétude de l'action publique. Simultanément, le chercheur en sciences sociales peut faire de cet engagement une opportunité d'observation des dynamiques et des processus, un mode de production de matériau de recherche, qu'il analysera avec plus de distance lorsqu'il refranchira le seuil et reprendra une place moins directement participante.

\section{RÉFÉRENCES BIBLIOGRAPHIQUES}

Alchian A., Demsetz H. (1973). The Property Right Paradigm. Journal of Economic History, vol. 33, $\mathrm{n}^{\circ}$ 1, pp. 16-27.

Apthorpe R. (1997). Writing development policy and policy analysis plain or clear. On langage, genre and power. In C. Shore, S. Wright (dir.), Anthropology of policy. London, Routledge, pp. 43-58.

Baré J.-F. (1995). La question des applications de l'anthropologie en France. In J.-F. Baré (dir.), Les applications de l'anthropologie: un essai de réflexion collective depuis la France. Paris, Karthala, pp. 9-23.

Béaur G., Schofield P., Chevet J.-M., PérezPicazo, M.-T. (dir.) (2013). Property rights, land markets and economic growth in the European countryside. Turnhout, Brepols.

Blondiaux L., Sintomer Y. (2009). L'impératif délibératif. Rue Descartes, vol. 63, $\mathrm{n}^{\circ} 1$, pp. 28-38.

Boone C. (2007). Property and constitutional order: Land tenure reform and the future of the African state. African Affairs, vol. 106, $\mathrm{n}^{\circ} 425$, pp. 557-586.

Borras Jr. S. M., Hall R., Scoones I., White B., Wolford W. (2011). Towards a better understanding of global land grabbing: an editorial introduction. Journal of Peasant Studies vol. $38, \mathrm{n}^{\circ}$ 2, pp. 209-216.

Callon M., Lascoumes P., Barthe Y. (2001). Agir dans un monde incertain. Essai sur la démocratie technique. Paris, Seuil, coll. « La Couleur des idées ».

Cefaï D. (1996). La construction des problèmes publics. Définitions de situations dans des arènes publiques. Réseaux, vol. $14, \mathrm{n}^{\circ} 75$, pp. 43-66.
Chauveau J.-P. (1998). La logique des systèmes coutumiers. In P. Lavigne Delville (dir.), Quelles politiques foncières pour l'Afrique rurale? Paris, Karthala/Coopération française, pp. 66-75.

Chauveau J.-P., Lavigne Delville P. (2013). Développement participatif. Dictionnaire critique interdisciplinaire de la Participation, http://www.participation-et-democratie.fr/fr/node/1313.

Colin J.-P. (2008). Disentangling intra-kinship property rights in land: a contribution of economic ethnography to land economics in Africa. Journal of Institutional Economics, vol. 4, n² 2, pp. 231-254.

Colin J.-P., Woodhouse P. (2010). Land markets in Africa. Edinburg, Edinburgh University Press.

Delmas C. (2011). Sociologie politique de l'expertise. Paris, La Découverte, coll. « Repères ».

De Singly F. (2002). La sociologie, forme particulière de conscience. In B. Lahire (dir.), À quoi sert la sociologie? Paris, La Découverte, pp. 13-42.

De Soto H. (2005). Le mystère du capital: pourquoi le capitalisme triomphe en Occident et échoue partout ailleurs. Paris, Flammarion.

Dubois V. (2003). La sociologie de l'action publique. De la socio-histoire à l'observation des pratiques (et vice-versa). In CURAPP (dir.), Historicités de l'action publique. Paris, Presses universitaires de France, pp. 347-364.

Dumoulin L. (2005). Présentation. Des modes de socialisation des savoirs académiques. Droit et société, vol. 60, n² 2, pp. 295-307. 
Fassin D. (2012). Sur le seuil de la caverne : l'anthropologie comme pratique critique. In P. Haag, C. Lemieux (dir.). Faire des sciences sociales. Paris, Éditions de l'EHESS, pp. 263-287.

Faure A., Pollet G., Warin P. (dir.) (1995). La construction du sens dans les politiques publiques. Débats autour de la notion de référentiel. Paris, L'Harmattan.

Filer C. (2006). Custom, Law and Ideology in Papua New Guinea. The Asia Pacific Journal of Anthropology, vol. 7, $\mathrm{n}^{\circ}$ 1, pp. 65-84.

Gaxie D. (1997). Luttes d'institutions : enjeux et contradictions de ladministration territoriale. Paris, L'Harmattan.

Hassenteufel P. (2010). Les processus de mise sur agenda: sélection et construction des problèmes publics. Informations sociales, vol. $157, \mathrm{n}^{\circ} 1$, pp. 50-58.

Jacob J.-P., Le Meur P.-Y. (2010). Citoyenneté locale, foncier, appartenance et reconnaissance dans les sociétés du Sud. In J.-P. Jacob, P.-Y. Le Meur (dir.), Politique de la terre et de l'appartenance. Droits fonciers et citoyenneté locale dans les sociétés du Sud. Paris, Karthala, pp. 5-57.

Jobert B. (1998). La régulation politique : le point de vue d'un politiste. In J. Commaille, B. Jobert (dir.), Les métamorphoses de la régulation politique. Paris, LGDJ, pp. 119-143.

Jobert B. (2004). Une approche dialectique des politiques publiques. L'héritage de l'État en action, Pôle Sud, vol. 21, n 2, pp. 43-54.

Kitching G. (1982). Development and Underdevelopment in Historical Perspective. London, Methuen.

Kuper A. (1982). Lineage Theory: A Critical Retrospect. Annual Review of Anthropology, vol. 11, pp. 71-95.

Lascoumes P. (2002a). De l'utilité des controverses socio-techniques. Journal international de bioéthique, vol. 13, $\mathrm{n}^{\circ} 2$, pp. 68-79.

Lascoumes P. (2002b). L'expertise, de la recherche d'une action rationnelle à la démocratisation des connaissances et des choix. Revue française d'administration publique, vol. 103, n 3, pp. 369-377.

Lascoumes P., Le Galès P. (2007). Sociologie de l'action publique. Paris, Armand Colin, coll. « $128 »$.
Lascoumes P., Simard L. (2011). L'action publique au prisme de ses instruments. Revue française de science politique, vol. $61, \mathrm{n}^{\circ} 1$, pp. 5-22.

Lavigne Delville P., Karsenty A. (1998). Des dynamiques plurielles. In P. Lavigne Delville (dir.), Quelles politiques foncières en Afrique noire rurale? Réconcilier pratiques, légitimité et légalité. Paris, Ministère de la Coopération/Karthala, pp. 215-242.

Lavigne Delville P. (2006). Sécurité, insécurités et sécurisation foncières: un cadre conceptuel. Réforme agraire et coopératives, $\mathrm{n}^{\circ} 2$, pp. 18-25.

Lavigne Delville P. (2007). À la recherche du chaînon manquant. Construire des articulations entre recherche en sciences sociales et pratique du développement. In T. Bierschenk, G. Blundo, Y. Jaffré, M. Tidjani Alou (dir.), Une anthropologie entre rigueur et engagement. Essais autour de l'cuvre de Jean-Pierre Olivier de Sardan. Leiden-Paris, APAD/Karthala, pp. 127-150.

Lavigne Delville P. (2009). Conceptions des droits fonciers, récits de politique publique et controverses. Les Plans fonciers ruraux en Afrique de l'Ouest. In J.-P. Colin, P.-Y. Le Meur, É. Léonard (dir.), Les politiques d'enregistrement des droits fonciers. Du cadre légal aux pratiques locales. Paris, Karthala, pp. 69-103.

Lavigne Delville P. (2010). La réforme foncière rurale au Bénin : émergence et mise en question d'une politique instituante dans un pays sous régime d'aide. Revue française de science politique, vol. 60, $\mathrm{n}^{\circ} 3$, pp. 467-491.

Lavigne Delville P. (2011). Vers une socio-anthropologie des interventions de développement comme action publique. Mémoire d'habilitation à diriger des recherches Université de Lyon II, Lyon, 210 p.

Leca J. (2012). L'État entre politics, policies et polity, ou peut-on sortir du triangle des Bermudes? Gouvernement et action publique, vol. 1, $\mathrm{n}^{\circ}$ 1, pp. 59-82.

Le Meur P.-Y. (2006). Governing Land, Translating Rights. The Rural Land Plan in Benin. In D. Mosse, D. Lewis (dir.), Development Brokers \& Translators. Ethnography of Aid and Agencies. Bloomsfield, Kumarian Press, pp. 75-99. 
Le Meur P.-Y. (2010). Réflexions sur un oxymore. Le débat du cadastre coutumier en Nouvelle-Calédonie. In E. Faugère, I. Merle (dir.), La Nouvelle-Calédonie, vers un destin commun? Nouveaux enjeux, nouveaux terrains. Paris, Karthala, pp. 101-126.

Le Meur P.-Y. (2011a). Politique et savoirs fonciers en Nouvelle-Calédonie: retour sur une expérience d'anthropologie appliquée, Journal de la société des océanistes, vol. 132, n 1, pp. 93-108.

Le Meur P.-Y. (2011b). Une petite entreprise de réassemblage du monde. Ethnographie et gouvernance des ressources foncières en Afrique de l'Ouest. Ethnologie française, vol. XVI, n 3 , pp. 431-442.

Li T. M. (2007). The Will to Improve. Governmentality, Development, and the Practice of Politics. Durham and London, Duke University Press.

Lund C. (2002). Negotiating property institutions: On the symbiosis of property and authority in Africa. In K. Juul, C. Lund (dir.), Negotiating property in Africa. Portsmouth, Heineman, pp. 11-43.

Massardier G. (2003). Politiques et action publiques. Paris, Armand Colin.

Moore S. F. (1973). Law and social change: the semi-autonomous social field as an appropriate subject of study. Law \& Society Review, vol. 7, n 4, pp. 719-746.

Muller P. (1990). Les politiques publiques. Paris, Presses universitaires de France, coll. «Que sais-je?».

Palier B., Surel Y. (2005). Les «trois I» et l'analyse de l'État en action. Revue française de science politique, vol. $55, \mathrm{n}^{\circ} 1$, pp. 7-32.

Platteau J. P. (1996). The Evolutionary Theory of Land Rights as Applied to Sub Saharan Africa: A Critical Assessment. Development and Change, vol. 27, $\mathrm{n}^{\circ}$ 1, pp. 29-86.

Pressman J. L., Wildavsky A. B.

Implementation. Berkeley, University of California Press.

Robert C. (2008). Expertise et action publique. In O. Borraz, V. Guiraudon (dir.), Politiques publiques. Tome 1. La France dans la gouvernance européenne. Paris, Presses de Sciences Po, pp. 309-335.

Roe E. (1994). Narrative Policy Analysis. Theory and Practice. Durham-London, Duke University Press.

Roqueplo P. (1997). Entre savoir et décision, l'expertise scientifique. Paris, INRA.

Sennett R. (2009). Nouveau capitalisme et expertise quotidienne. Cahiers internationaux de sociologie, vol. 126, pp. 13-20.

Stamm V. (2013). Formaliser les pratiques coutumières. Europe médiévale, Afrique coloniale et contemporaine. Études rurales, vol. 191, n 1 , pp. 169-189.

Théry I. (2005). Expertises de service, de consensus, d'engagement: essai de typologie de la mission d'expertise en sciences sociales. Droit et société, vol. $60, \mathrm{n}^{\circ} 2$, pp. 311-327.

Zittoun P. (2013). La fabrique politique des politiques publiques: une approche pragmatique de l'action publique. Paris, Presses de Sciences Po, coll. «Sciences Po. Gouvernances ». 